\title{
Quand les protéines virales \\ s'associent au complexe majeur d'histocompatibilité
}

L.cs virus sont des micro-organismes intraccllulaires obligatoircs dont la prolifération est en général combattuc par les anticorps et les lymphocytes $\mathrm{T}$ cytotoxiques CD8 qui ćliminent les cellules infectécs. La pathologic provoquéc par certains virus peut être directement lićc à la dégradation des tissus infectés. Les lymphocytes T CD8 reconnaissent des peptides viraux présentés par les molćcules de classe I du complexc majcur d'histocompatibilité (CMH-I) [1]. Ces peptides proviennent de la dégradation des protéines virales dans le cytoplasme et sont transloqués dans le réticulum endoplasmique par des transporteurs. Les complexes peptide-CMH-I suivent la voie classique d'cxocytose pour atteindre la surface ccllulaire. La multiplication des allc̀les et des loci rend trc̀s improbable l'absence de présentation des antigc̀ncs viraux par les molécules CMH-I. Presque toutes les cellules cxpriment lc CMH-I et sont donc soumiscs à la survcillance immunologique effectuée par les lymphocytes $\mathrm{T}$ cytotoxiques. Si l'élimination des cellules infectées a lieu cssentiellement par l'intermédiaire des lymphocytes T CD8, le rôle des lymphocytes $\mathrm{T}$ auxiliaircs CD4 pcut ĉtre déterminant en fonction du mode d'infection, de la voic d'entrée du virus dans la cellule et de la distribution tissulaire des cellules présentant l'antigène. Les lymphocytcs T CD4 reconnaissent les peptides antigéniques présentés par les molécules de classe II du CMH (CMH-II) [1]. I.es peptides présentés aux lymphocytcs T CD4 sont, en général, produits dans les endosomes (ou lysosomes) et résultent de la dégradation d'antigc̀nes exogènes internalisés dans la voie d'cndocytose. Le transport des molécules de classe II $\mathrm{du} \mathrm{CMH}$ et leur interaction avec les peptides sont en partie réglés par la chaîne invariante li qui est leur protéine chaperon [2-4]. biologiques qui lui sont spécifiques et dont certaincs favorisent le détournement de la réponsc antivirale cellulaire ct immunitaire (voir [5-7] pour revue). Les virus ont, par exemple, les moyens de contrôler certaincs fonctions du complément, des interférons et du facteur nécrosant les tumcurs. Ils synthétisent des inhibiteurs et des homologues de cytokines et peuvent interférer avec la présentation antigénique en agissant sur les mécanismes de dégradation des protéincs cytosoliques. Les niveaux d'cxpression du CMH et des molécules d'adhérence sont fréquemment modifiés à la suite d'infections virales d'origine diversc. Cette synthèse n'a pas la prétention de dresser unc liste exhaustive des mécanismes de détournement de la réponse antivirale mais plutôt d'en présenter quelques exemples dont le caractère commun est lié à l'intcraction directe de protéines virales avec les molécules présentatriccs d'antigènes (Tableau I).

Associations des protéines virales au CMH-I

La modulation de l'expression du CMH-I par les virus est un phénomène fréquemment observé qui peut provenir du contrôle de la transcription des gènes du CMH-I. Les deux exemples présentés ci-dessous montrent que des virus peuvent aussi influencer la reconnaissance des cellules infectécs par les lymphocytes T CD8 en inhibant le transport des molécules CMH-I vers la surface cellulaire.

Les adénovirus humains (Ad) sont la cause d'infections respiratoires qui peuvent être persistantes. La région E3 de l'Ad2 code pour la glycoprotéine précoce transmembranaire E19, qui présente deux caractéristiques essentielles [8]. Elle peut s'associcr à la chaîne lourde du CMH-I (sous-unité $\alpha$ ) par sa portion luminale. Les mutants de délétion de E19 ayant perdu les régions intracytoplasmique et transmembranairc gardent leur capacité à s'associer à la chaîne $\alpha$ du CMH-I. La scconde caractéristique de cctte protéine viralc est sa localisation intracellulairc. E19 est strictement une protéine du réticulum endoplasmique mais les mutants de délétion sont sécrétés par la voic classique. En fait, les 15 acides aminćs intracytoplasmiques de E19 contiennent une séquence de rétention dans le réticulum endoplasmique qui reste fonctionnelle lorsqu'elle est transférée sur une autre protéine. La protéine E19 peut donc former des complexcs avec les molécules du CMH-I nouvellement synthétisées et, par l'intermédiaire de sa région intracytoplasmique, empêcher leur

\begin{tabular}{|c|c|c|c|}
\hline \multicolumn{4}{|c|}{$\begin{array}{l}\text { Tableau I } \\
\text { CTIONS DE PROTÉINES VIRALES } \\
\text { PROTÉINES DE L'HÔTE }\end{array}$} \\
\hline Virus & Protéines virales & Protéines de l'hôte & Conséquences \\
\hline $\begin{array}{l}\text { Ad-2 } \\
\text { HCMV } \\
\text { HSV-1 } \\
\text { MMTV } \\
\text { Rage }\end{array}$ & $\begin{array}{l}\text { E3-gp 19K } \\
\text { UL18 } \\
\text { nd } \\
\text { 3'ORF } \\
\text { Nucléoprotéine }\end{array}$ & $\begin{array}{l}\mathrm{CMH}-\mathrm{l} \alpha \\
\beta 2 \mathrm{~m} \\
\text { nd } \\
\mathrm{CMH}-\mathrm{II} \\
\mathrm{CMH}-\mathrm{Il}_{\alpha}\end{array}$ & $\begin{array}{l}\text { Transport CMH-I } \\
\text { Transport CMH-I } \\
\text { Transport CMH-II } \\
\text { Superantigène } \\
\text { Superantigène }\end{array}$ \\
\hline
\end{tabular}

Ces interactions ont un effet inhibiteur sur le transport des CMH-I et CMH-II, et permettent le déclenchement d'une activité virale superantigénique (voir détails dans le texte). 
transport hors du réticulum endoplasmique vers l'appareil de Golgi et la surface [9]. Il en résulte une diminution de la densité de surface du $\mathrm{CMH}$ I et une faible sensibilité des cellules exprimant E19 à la cytotoxicité des lymphocytes T CD8 [10].

Les infections par le cytomégalovirus humain (HCMV) peuvent avoir des conséquences graves, en particulier chez les sujets immunodéprimés. Le génome viral code pour une protéine UL 18 homologue de la chaîne lourde du CMH-I (sous-unité $\alpha$ ) des eucaryotes supérieurs [11, 12]. Comme la sous-unité $\alpha$ du CMH-I, la protéine virale UL18 s'associe à la $\beta 2$-microglobuline $\left(\beta_{2} \mathrm{~m}\right)$. La $\beta 2 \mathrm{~m}$ est une protéine soluble qui s'apparie avec la chaîne lourde CMH-I pour former un complexe dont la structure est stabilisée par la fixation de peptides dans le réticulum endoplasmique. L'infection par HCMV conduisant à une diminution importante de l'expression de surface du CMH-I, il est possible que la compétition, entre UL18 et la chaîne lourde du CMH-I, pour l'association avec la $\beta 2 \mathrm{~m}$ soit très en faveur de la protéine UL18. Le transport de la chaîne $\alpha$ du CMH-I étant dépendant de son assemblage avec la $\beta 2 \mathrm{~m}$, l'expression de surface des molécules du CMH-I serait liée à la quantité de $\beta 2 \mathrm{~m}$ libre dans le réticulum endoplasmique, non piégée par UL18. Le fait que la cytotoxicité anti-HCMV soit principalement dirigée contre des protéines synthétisées immédiatement après l'infection est peut-être à rapprocher de ce phénomène. L'inhibition de la maturation des molécules CMH-I n'est cependant pas la seule stratégie employée par le CMV pour se protéger des défenses immunitaires de l'hôte. En effet, l'infection par le CMV murin n'affecte pas le niveau d'expression du CMH-I, mais la présentation de l'antigène viral immunodominant est sélectivement inhibée [13]. De plus, la paralysie de la voie endogène de présentation de l'antigène (lymphocytcs T CD8/CMH-I) n'est probablement pas suffisante pour bloquer les défenses cytotoxiques de l'hôte. Cela a été montré, dans un système il est vrai très différent, lorsquc des souris déficientes pour la $\beta 2 \mathrm{~m}$ sont infectées par le virus de la chorio- méningite lymphocytaire [14]. Les souris $\beta 2 \mathrm{~m}^{-1-}$ n'expriment pas les molécules CMH-I en surface et ne produisent pas un nombre significatif de lymphocytes T CD8. Chez ces souris, l'absence de lymphocytes T CD8 conduit au développement d'une réponse cytotoxique des lymphocytes T CD4

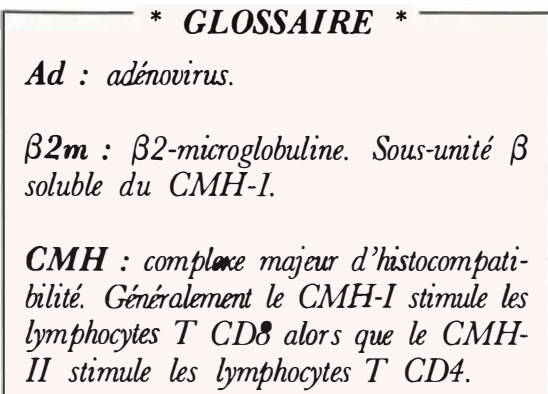

DR, DQ DP : isotypes du CMH-II.

$\boldsymbol{E} \boldsymbol{B} \boldsymbol{V}$ : virus d'Epstein-Barr.

E3-gp 19K : protéine de l'Ad2 qui s'associe à la sous-unité $\alpha$ du CMH-I et bloque son transport.

HCMV : cytomégalovirus humain.

HSV-1 : virus Herpes simplex 1.

MMTV : virus des tumeurs mammaires murines (mouse mammary tumor virus).

MuLV: virus leucémogène murin (murine leukemia virus).

3'ORF : phase de lecture ouverte dans les séquences répétées 3' du MMTV (3' open reading frame).

\section{$\mathrm{SAg}$ : superantigène.}

UL18 : protéines de HCMV qui s'associent avec la $\beta 2 \mathrm{~m}$.

$V \beta$ : région variable de la sous-unité $\beta$ $d u$ récepteur $T$. Site d'interaction avec les superantigènes.

$\boldsymbol{V I H}$ : virus de l'immunodéficience humaine.

VIS : virus de l'immunodéficience simienne. stimulés par le CMH-II. Des clones cytotoxiques T CD4 ont d'ailleurs été décrits chez la souris infectée par les virus influenza et de la stomatite vésiculeuse ainsi que chez l'homme à la suite d'infection par les virus influenza, de la rougeole et de l'Herpes simplex (HSV) et après vaccination contre la rage.

\section{Associations des protéines virales au} CMH-II

L'interaction des molécules du CMHII avec des protéines virales a été démontrée lors de la découverte des superantigènes viraux $[15,16]$. Les superantigènes (SAg) sont d'origine bactérienne ou virale et diffèrent des antigènes conventionnels pour au moins quatre raisons. La présentation des SAg au système immunitaire est la conséquence de leur association aux molécules du CMH-II, qui n'implique pas le site de fixation au peptide. La présentation est dite non restreinte car le polymorphisme du CMH-II n'a que peu d'influence sur l'association avec les SAg. Les SAg sont présentés aux lymphocytes $\mathrm{T}$ sous leur forme native, sans dégradation préalable en peptides. Enfin, la stimulation des lymphocytes $\mathrm{T}$ est la conséquence de l'interaction du SAg avec les séquences variables de la sous-unité $\beta$ du récepteur T $(\mathrm{V} \beta)$. La stimulation est donc indépendante de la spécificité antigénique du lymphocyte $T$, qui fait intervenir les deux sous-unités du récepteur ( $\alpha$ et $\beta$ ). Il résulte de ce mode d'interaction qu'une stimulation superantigénique recrute 1000 à 10000 fois plus de lymphocytes $\mathrm{T}$ qu'une réponse à un antigène conventionnel. Une hyperstimulation incontrôlée du système immunitaire, provoquée par une protéine virale superantigénique, empêcherait le développement d'une réponse antivirale spécifique. L'action des SAg sur le répertoire $T$ peut aussi se traduire par la délétion ou l'inactivation des lymphocytes $\mathrm{T}$ porteurs de $\mathrm{V} \beta$ cible. Ces caractéristiques des SAg font que leur contribution a été suggérée dans certaines maladies auto-immunes et l'immunodéficience acquise. Le premier SAg viral a été décrit chez les souris infectées par le virus des tumeurs mammaires (MMTV) [17, 18]. Le SAg du MMTV est une pro- 
téine transmembranaire codée par une phase ouverte de lecture de la séquence répétée 3' (3'ORF). Une activité superantigénique virale a été suggéréc mais non démontrée pour expliquer le désordre immunitaire provoqué par les virus leucémiques murins (MuLV) [19]. Dans le cas des rétrovirus murins, il a été proposé que la prolifération cellulaire, induite par le SAg, puisse favoriser l'incorporation virale dans le génome de l'hôte. Chez l'homme, le seul SAg viral identifić jusqu'à présent est la nucléoprotéine du virus de la rage [16]. Cette protéine interne s'associe à la chaîne $\alpha$ du $\mathrm{CMH}-\mathrm{II}$ et stimule la prolifération des lymphocytes $\mathrm{T} V \beta 8$. D'autres SAg seront probablement isolés à partir de virus induisant une prolifération lymphocytaire suivie d'une immunodéficience plus ou moins forte et prolongée. Les virus fréquemment cités qui entrent dans cette catégorie sont le virus de la rougeole, l'EBV, le CMV et le VIH.

La survic de l'hôte infecté par le virus Herpes simplex 1 (HSV-1) passe par une immunité spécifique à médiation ccllulaire qui maintient le virus sous la forme d'une infection latente du système nerveux périphérique et contral. Si l'infection latente ne peut pas être établie, le virus envahit le système nerveux et provoque la mort de l'hôte. C'est le cas en particulier lorsque le virus infectant est de la souche virulente $\mathrm{KOS}$ ou lorsque la souche $\mathrm{F}$, non virulente pour des souris normales, devient létale pour des souris immunodéficientes. Quel que soit le niveau de virulence de la souche virale, les lymphocytes T CD4 et CD8 sont attirés vers les sites de l'infection, et l'expression des molécules de classe II du CMH est induite [20]. Cependant, contrairement à ce qui est observé lors d'une infection par la souche $\mathrm{F}$ non virulente, les protéines de classe II du $\mathrm{CMH}$ exprimées par les cellules de la microglie ne sont pas transportées en surface lorsque l'induction est la conséquence d'une infection par la souche virulente $\mathrm{KOS}$. Les raisons de la séquestration intracellulaire des complexes de classe II du CMH peuvent être multiples. Par analogic avec le HCMV et l'Ad2, une protéine de HSV-1 pourrait bloquer la formation de l'hétérotrimc̀re en s'associant avec une des trois sous-unités $(\alpha, \beta$, Ii). Il faut rappeler que des anomalies de transport des complexes de classe II du $\mathrm{CMH}$ ont été récemment décrites chez les souris déficientes pour la chaîne Ii qui est la molécule chaperone des dimères $\alpha \beta$ ([21] et $m / s n^{\circ} 3$, vol. 9, p. 350). La synthèse ou la fonction de la chaîne Ii est-elle modifiéc lors de l'infection par une souche virale virulente? $\mathrm{Si}$ la composante CD4 est essentielle au développement d'une réponse antivirale performante, la virulence de la souche KOS pourrait s'expliquer, en partic, par l'absence de présentation des peptides viraux par les molécules de classe II du CMH. Un rapprochement prudent pourrait être fait avec l'immunodéficience induite par HCMV et VIH si un mécanisme d'inhibition de présentation d'antigènes était observé à la suite de l'infection des macrophages ou des cellules dendritiques. Quoi qu'il en soit, comme pour les virus précédemment cités, la maîtrise de l'expression des molécules du CMH n'est pas la seule stratégie employée par HSV-1 pour échapper à la surveillance immunologique. Il est probable que cet échappement résulte d'un large déploiement d'astuces destinées à contrôler des fonctions auss essentielles que la présentation antigénique, l'activation des voies du complément et la phagocytose.

\section{Présence du CMH sur les particules virales}

Les virus possèdent à leur surface des récepteurs pour les composants de l'hôte. Ces récepteurs sont parfois identifiés par leurs ligands naturels. Un des ligands de HCMV libre est la $\beta 2 \mathrm{~m}$ [22]. Dans ce cas, la chaîne lourde CMH-I n'est pas présente sur le virus et il est possible, mais non prouvé, que le récepteur viral de la $\beta 2 \mathrm{~m}$ soit la protéine UL18 décrite plus haut. Les conséquences physiologiques de l'association de la $\beta 2 \mathrm{~m}$ avec le virus ne sont pas élucidées clairement, mais le virus libre associé à la $\beta 2 \mathrm{~m}$ dans les liquides biologiques pourrait être moins sensible à la neutralisation par les anticorps anti-HCMV. Il a aussi été suggéré que la présence de la $\beta 2 \mathrm{~m}$ sur le virus pourrait faciliter l'infection en favorisant la fixation du virus sur les cellules par l'intermédiaire des chaînes lourdes CMH-I de l'hôte. En relation avec ce phénomène, il est intéressant de noter que les cellules infectées produisent de l'interféron $\gamma$, qui induit une augmentation de l'expression du $\mathrm{CMH}-\mathrm{I}$ et de la sécrétion de $\beta \mathrm{m} 2$ par les cellules saines.

Les virus et en particulier les rétrovirus associent fréquemment des protéines cellulaires dans leur enveloppe. Le cas du virus d'immunodéficience humaine (VIH) est particulièrement intéressant car il montre une incorporation sélective des protéines du $\mathrm{CMH}$ dans l'enveloppe virale. En effet, parmi une panoplic d'anticorps monoclonaux dirigés contre les protéines de surface d'une lignée $\mathrm{T}$ productrice du VIH, seulement les anticorps anti- $\beta 2 \mathrm{~m}$, anti$\mathrm{CMH}-\mathrm{II}$ et à un moindre niveau antiCMH-I réagissent avec les lysats viraux [23]. Plus récemment, les protéines cellulaires associées au VIH et au VIS (virus d'immunodéficience simicnne) ont été purifiées et identifiées [24]. Il s'agit majoritairement de l'actine, de l'ubiquitine, de la $\beta 2 \mathrm{~m}$ et d'un isotype CMH-II (DR). La très faible quantité de chaîne lourde $\mathrm{CMH}$ I et l'absence des autres isotypes CMH-II (DQ et DP) montrent le caractère très sélectif de l'incorporation des protéines cellulaires dans l'enveloppe virale. Le rôle des protéines du $\mathrm{CMH}$ sur les particules virales n'est pas élucidé. Comme pour le HCMV, il est possible que ces protéines cellulaires facilitent l'infection en augmentant le pouvoir d'adhérence du virus. D'autre part, la présence du CMH-II sur le virus pourrait avoir des conséquences importantes pour le système immunitaire. En effet, en fonction de l'état de maturation des lymphocytes $\mathrm{T}$ et de la capacité des cellules présentant l'antigène à transmettre des signaux d'activation, la formation du complexc récepteur T-peptide-CMH-II peut conduire à l'activation, à l'anergie ou à l'apoptose des lymphocytes T. Or, en théorie, les virus sont recouverts d'un nombre suffisant de complexes CMHII pour être reconnus par les lymphocytes T CD4. Cette reconnaissance, non couplée aux signaux d'activation, pourrait-elle jouer un rôle dans l'immunodéficience ? L'association des molécules DR du CMH-II avec 
l'envcloppe virale a-t-elle lieu au hasard ou cst-cllc dépcndante des peptides antigéniques qu'elles pourraicnt présenter?

Une caractéristique du système immunitaire est son pouvoir d'adaptation très puissant à des événements nouveaux. Les relations hôte-virus sont donc établies sur un mode dynamique et font appcl à des mécanismes biologiques fondamentaux qui offrent aux médecins ct aux cherchcurs un moyen d'étude inestimable de la biologie de la cellule, du virus et du système immunitaire

\section{Vincent Lotteau}

Inserm CJF 8804, Institut biomédical des Cordeliers, Immunogénétique, 15, rue de l'École de Médecine, 7.5006 Paris, France.

\section{RÉFÉRENCES}

1. Brodsky FM, Guagliardi L.E. Ihe cell biology of antigen processing and presentation. Annu Rev Immunol 1991; 9 : 707-44.

2. 'T'cyton I, O'Sullivan D, Dickson P, Lotteau V, Sette A, Fink P, Peterson PA. Invariant chain distinguishes the exogenous and the cndogenous antigen presentation pathways. Nature $1990 ; 348: 39-44$

3. I ottcau V, Teyton I, Peleraux A, Nilsson 'T', Karlsson I, Quaranta V, Schmid S, Peterson PA. Invariant chain directs intracellular trafficking of class II MHC molecules. Nature 1990 ; 348: 600-5

4. Rabourdin-Combe C, Bertolino P, CalinLaurcns V, Gerlicr D. I a présentation de l'antigène aux lymphocytes T. médecine/sciences $1991 ; 7$ : 674-80.

5. Gooding I R. Virus proteins that counteract host immune defenses. Cell $1992 ; 71$; 5-7.

6. Maudsley JD, Pound JD. Modulation of $\mathrm{MHC}$ antigen expression by viruses and oncogenes. Immunol Today 1991; 12 : 429-31.

7. Driscoll J, Finley D. A controlled breakdown : antigen processing and the turnover of viral proteins. Cell $1992 ; 68: 823-5$.

8. Pääbo S, Wcber F, Kämpe O, Schaffner W, Peterson PA. Association between transplantation antigens and a viral membranc protein synthesized from a mammalian expression vector. Cell 1983 ; 33 : 445-53.

9. Pääbo S, Bhat BM, Wold WS, Peterson PA. A short scquence in the $\mathrm{COOH}$ tcrminus makes an adcnovirus membrane glycoprotein a resident of the cndoplasmic reticulum. Cell 1987; 50: 311-7.

$\mathrm{m} / \mathrm{s} \quad n^{\circ}$ 6-7 vol. 9, juin-juillet 93
10. Burgert HG, Maryanski J, Kvist S "E.3/19K » protein of adenovirus type 2 inhibits lysis of cytolytic $\mathrm{T}$ lymphocytes by blocking coll surface expression of histocompatibility class I antigens. Proc Natl Acad Sci USA 1987 ; 84 : 1356-60.

11. Browne H, Smith G, Beck S, Minson T. A complex between the MHC class I homologue cncoded by human cytomegalovirus and B2 microglobulin. Nature 1990; 347 : 770-2. 12. Beck S, Barrcll BG. Human cytomegalovirus encodes a glycoprotein homologous to MHC class I antigens. Nature 1988 ; 331 : 269-72.

13. Del Val $M$, Münch $K$, Reddchasc $M J$, Koszinowski UH. Presentation of CMV immediate early antigen to cytolytic $\mathrm{T}$ lymphocytes is sclectivcly prevented by viral genes cxpressed in the early phasc. Cell 1989 ; 58 : 305-15.

14. Muller D, Koller BH, Whitton I, IaPan KE, Brigman KK, Frelinger JA. I,CMVspecific class II restricted cytotoxic $T$ cells in $\beta 2$ microglobulin deficient micc. Science 1992 ; $255: 1576-8$

15. Acha-Orbea H, Palmer E. Mls : a retrovirus exploits the immunc system. Immunol Today 1991; 12 : 356-61.

16. Lafon M, I afage M, Martinez-Arends A, Ramircz R, Vuillier F, Charron D, Lottcau V, Scott-Algara D. Evidence for a viral superantigen in humans. Nature 1992 ; 358 : 507-9. 17. Choi Y, Kapler JW, Marrack P. A superantigen cncoded in the open reading frame of the 3' long terminal repcat of mouse mammary tumour virus. Nature $1991 ; 350$ : 203-7. 18. Acha-Orbea H, Shakhov AN, Scarpellino I, Kolb E, Müller V, Vessaz-Shaw A, Fuchs R, Blëchlinger K, Rollini P, Billotte J, Sarafidou M, Macl Donald HR, Iiggclmann $\mathrm{H}$. Clonal delction of $V \beta 14$ bearing $T$ cells in micc transgenic for mammary tumour virus. Nature 1991; 350: 207-11.

19. Hügin AW, Vacchio MS, Morsc HC. A virus encoded "superantigen" in a retrovirusinduced immunodeficiency syndrome of micc. Science $1991 ; 252$ : 424-7.

20. I ewandowski GA, L,o I), Bloom FE. Interference with major histocompatibility complex class II restricted antigen presentation in the brain by Herpes simplex virus type 1 : a possible mechanism of evasion of the immune response. Proc Natl Acad Sci USA $1993 ; 90: 2005-9$

21. Viville $S$, Neefjes J, I otteau V, Dicrich A, Lemeur $\mathrm{M}$, Ploegh $\mathrm{H}$, Benoist $\mathrm{C}$, Mathis D. Mice lacking MHC class II associated invariant chain. Cell 1993; 72 : 1-20. 22. Grundy JE. Alterations of cellular proteins in human after cytomegalovirus infection : potential for disease pathogenesis. Transplant Proc 1991 ; 23 : 38-42.

23. Hoxic JA, Fitzharris 'IP, Yougbar PR, Matthews DM, Rackowski JL, Radka S. Nonrandom association of cellular antigens with HTI,V III virions. Hum Immunol 1987 ; $18: 39-52$.

24. Arthur I.O, Bess JW, Sowder II RC, Benveniste RE, Mann DI, Chermann JC, Henderson I.E. Cellular proteins bound to immunodeficiency viruses: implications of pathogenesis and vaccincs. Science $1992 ; 258$ : 1935-8. 